\title{
Turbulence modelling of the flow past a pitching NACA0012 airfoil at $10^{5}$ and $10^{6}$ Reynolds numbers
}

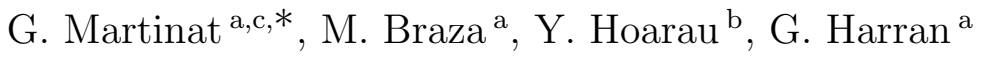 \\ ${ }^{a}$ Institut de mécanique des fluides de Toulouse, Allée Camille de Soula,31000, Toulouse, France \\ ${ }^{\mathrm{b}}$ Intitut de mécanique des fluides et solides de Strasbourg \\ ${ }^{\mathrm{c}}$ Center for Coastal and Physical Oceanography, Old Dominion University, Norfolk, Virginia
}

Received 18 February 2008; accepted 3 august 2008

\begin{abstract}
This paper provides a study of the NACA0012 dynamic stall at Reynolds numbers $10^{5}$ and $10^{6}$ by means of two and three dimensional numerical simulation. The turbulence effect on the dynamic stall is studied by statistical turbulence modelling. The results are compared with experiments concerning each test case. Standard URANS turbulence modelling have shown a quite dissipative character that attenuates the instabilities and the vortex structures related to the dynamic stall. The URANS approach OES, Organised Eddy Simulation, has shown an improved behaviour at the high Reynolds number range. Emphasis is given to the physical analysis of the three dimensional dynamic stall structure, for which there exist few numerical results in the literature, as far as the Reynolds number range is concerned. This study has shown that the downstroke phases of the pitching motion are subjected to strong three-dimensional turbulence effects along the span, whereas the flow is practically two-dimensional during the upstroke motion.
\end{abstract}

\section{Introduction}

The prediction of dynamic stall phenomenon at high Reynolds number is a crucial need in aeronautics and more specifically in rotorcraft dynamics. In this context, the forced unsteadiness (organised motion) interacts non-linearly with the fine-scale random turbulence and produces a strong irreversibility effect that usually leads to hysteresis loops in the aerodynamics coefficients versus the angle of incidence. This hysteresis effect is the result of a phase lag between the bodys motion and the near-region velocity-vorticity gradients. Under these above effects, the stall angle is found higher than the normal static stall angle. The dynamic stall creates conditions of strong non-equilibrium turbulence, where the turbulence kinetic energy production is not equal to the dissipation. In this case, standard turbulence modelling approaches are often insufficient to capture correctly the hysteresis effect and to predict the dynamic stall loads at high Reynolds number. The applications of these flows occur in turbomachinery and in helicopter rotorblades as well as in wind turbine airfoils. It is important to have a good prediction of the dynamic stall to ensure efficiency for design. A comprehensive review of the dynamic stall is described in McCroskey (1981, 1982), Chandrasekhara and Carr (1990) and Piziali (1993). Experimental work from Laboratoire d'Aérodynamique and Biomécanique du Mouvement (LABM) has given a detailed physical

\footnotetext{
* Corresponding author.

E-mail address: martinat@ccpo.odu.edu (G. Martinat).
} 
analysis of this phenomenon (Berton et al., 1997). More recently, Tsang et al. (2008) provides an experimental study of dynamic stall using force measurement and Akbari and Price (2003) provides a numerical study using vortex method to model the flow past a pitching airfoil.

In the context of a pitching airfoil, with a sufficiently high incidence angle that depends on flow parameters such as pitching amplitude, pitching frequency, average incidence and profile geometry, a high energy vortex develops on the leading edge of the profile and is advected near wall towards the trailing edge. Consequently, this vortex grows and leads to a boundary layer thickness increase and finally to separation. When the leading edge vortex is shed, the flow is fully stalled inducing a abrupt loss in the lift coefficient, an increase of drag coefficient and a negative peak on the moment coefficient. With the decrease of incidence, the flow finally reattaches from the leading edge.

From the point of view of numerical modelling, the work of the european program UNSI (Unsteady Viscous Methods in the Context of Fluid-Structure Interaction, Haase et al. (2002)) reports a comparison of CFD approaches for flows around pitching airfoils at high Reynolds number based on Piziali (1993) and Berton et al. (1997) experiments. It is reported that stantard URANS methods are unable to properly capture experimental hysteresis cycle. A slight improvement can be achieved by using non-linear eddy viscosity modelling (NLEVM), explicit algebraic Reynolds stress model [EARSM, Wallin and Johansson (2000)] or by calibrating a one-equation model based on the eddy viscosity transport equation of Spalart and Allmaras (1992) model, specifically for a pitching airfoil [SALSA model, Rung et al. (2002)]. Finally, Benyahia et al. (2003) have shown an improved behaviour by using $k-\omega$ SST model.

The aim of the present study is to evaluate the turbulence modelling performance by comparing classical and advanced URANS approaches in two- and three-dimensional simulation. Furthermore, dynamic stall is a peculiar case, less dependant on three-dimensional effects than "static" bluff body turbulence during the upstroke phase and more dependant on three-dimensionality during the downstroke phase (Berton et al., 2002). Therefore, an emphasis is given is given on three-dimensional simulation of the dynamic stall at high Reynolds number. Hybrid (URANS-LES) turbulence modelling is employed in this case by using a DDES, Delayed Detached Eddy Simulation [DDES, Spalart et al. $(1997,2006)]$. The results are compared ith two-dimensional statistical turbulence modelling.

\section{Turbulence modelling : macrosimulation for unsteady flows}

\subsection{Organised Eddy Simulation (OES)}

Advanced URANS modelling aims at capturing non-equilibrium turbulence effects especially occuring in the dynamic stall context. Among different macrosimulation approaches, the Organised Eddy Simulation (OES) (Braza et al. (2006)) was developed by the research group EMT2 (Ecoulements Monophasiques Transitionnels et Turbulents) at CNRS/IMFT (Centre National de la Recherche Scientifique, Institut de Mécanique des Fluides de Toulouse). This approach consists of distinguishing the structures to be resolved from the structures to be modelled on the basis of their physical nature, organised or chaotic and not on their size as in LES approach. According to OES, the turbulence spectrum is extended in whole the range from low to high wavenumbers and therefore it can be modelled by statistical turbulence modelling consideration. However, due to strong non-equilibrium turbulence effects in the present class of inhomogeneous turbulence flows, shape and slope modification occur in the inertial range of the turbulence energy spectrum, as shown in detailed experimental studies by Braza et al. (2006) using time-resolved PIV. As a consequence, production is not equal to dissipation like in URANS equilibrium turbulence modelling. Therefore, the statistical turbulence scales used to model the continuous part of the energy spectrum have to be properly reconsidered to capture the non-equilibrium effects. In OES, the organised part of the motion corresponds to distinguished peaks in the spectrum that correspond to the resolved turbulence. In the time-domain, due to the pronounced periodic character of the pitching motion (forced unsteadiness), the phase-averaged Navier-Stokes equations are considered according to the decomposition of Cantwell and Coles (1983). The periodic nature of the flow past an oscillating airfoil allows definition of phase-averaged quantities. The flow is decomposed into a periodic component and a random fluctuation : $\left\langle U_{i}\right\rangle=\bar{U}_{i}+\tilde{U}_{i}$.

A detailed presentation of the OES approach is provided in a companion paper by Bourguet et al., in the present dedicated volume of the the IUTAM Symposium "Unsteady Separated Flows and their Control". The reader is kindly invited to refer to this companion paper for more details concerning this approach. OES has been applied succesfully in a number of European aeronautics research programs, among which, ETMA [Efficient Turbulence 
Models in Aeronautics, Dervieux et al. (1998)], FLOMANIA [Flow Physics Modelling an Integrated Approach, Bouhadji et al. (2002)] and DESIDER [Detached Eddy Simulation for Industrial Aerodynamics].

In the present study, a first-order OES modelling is employed (Bourdet et al., 2007), derived from a second order moment closure DRSM (Launder et al., 1975). In this context, a modified two-equation model was derived, where the turbulence length scales were modified by means of evaluation of the $C_{\mu}$ eddy diffusion coefficient through DRSM and by reconsidering the turbulence damping law towards the wall (Hoarau et al., 2002; Jin and Braza, 1994). In the context of high-order stress-strain modelling, a tensorial OES eddy-viscosity model has been derived to capture the non-equilibrium turbulence (Bourguet et al., 2007) where the $C_{\mu}$ eddy diffusion coefficient varies according to a directional criterion of stress-strain misalignment. The first order OES modelling yields a two-equation low-Reynolds that derives from the original low-Reynolds two-equation model from Chien (1982). the present model is proven less dissipative than the standard two-equation first-order models and is described by the following system of equations :

$$
\begin{gathered}
\frac{D k}{D t}=\frac{\partial}{\partial y}\left[\left(\nu+\nu_{t}\right) \frac{\partial k}{\partial x_{j}}\right]+\nu_{t}\left(\frac{\partial \overline{U_{i}}}{\partial x_{j}}\right)^{2}-\epsilon-\frac{2 \nu k}{y^{2}} \\
\frac{D \epsilon}{D t}=\frac{\partial}{\partial x_{j}}\left[\left(\nu+\frac{\nu_{t}}{\sigma}\right) \frac{\partial \epsilon}{\partial x_{j}}\right]+C_{1} \frac{\epsilon}{k} \nu_{t}\left(\frac{\partial \overline{U_{i}}}{\partial x_{j}}\right) 2-\frac{\epsilon}{k}\left[C_{2} f_{\epsilon}+\frac{2 \nu k \mathrm{e}^{-C_{4} \frac{U_{*} y}{\nu}}}{y^{2}}\right]
\end{gathered}
$$

where,

$$
\begin{gathered}
f_{\epsilon}=1-\frac{0.4}{1.8} \exp \left(-\left(k^{2} / 6 \nu \epsilon\right)^{2}\right), \\
\nu_{t}=C_{\mu} f_{\mu} \frac{k^{2}}{\epsilon}
\end{gathered}
$$

with

$$
\begin{gathered}
C_{\mu}=0.02 \\
f_{\mu}=1-\exp \left(0.0002 y^{+} 0.000065 y^{+2}\right) .
\end{gathered}
$$

The advantages of this approach are the robustness at high Reynolds number wall bounded flows and the fact that the method is not intrinsically three-dimensional.

\subsection{Detached Eddy Simulation (DES)}

A reported by Travin et al. (2000), the Detached-Eddy Simulation is a three-dimensional simulation using statistical turbulence modelling equations, where the turbulence length scale is chosen between its RANS expression and a sub-grid length scale as in Large Eddy Simulation. This choice operates as a RANS model in the near-wall region and as a LES model in the regions of flow detachment. The advantage of the present hybrid (RANS-LES) approach comparing to other hybrid approaches is that it uses a single set of statistical turbulence modelling equations and does not require the definition of an interface between RANS and LES regions. The DES approach is intrinsically 3 -D.

The DES length scale is chosen according to the following equation :

$$
\tilde{d}=\min \left(d, C_{D E S} \Delta\right),
$$

where $C_{D E S}$ is the DES constant calibrated by means of homogeneous, isotropic turbulence spectrum, $\Delta$ is the largest dimension of the elementary control volume cell, $\Delta=\max (\Delta x, \Delta y, \Delta z)$ and $d$ is the URANS lengthscale. This is the distance to the nearest wall in the case of Spalart and Allmaras modelling and a distance homogeneous to $k^{3 / 2} / \epsilon$ in two equation modelling. For example, for a $k-\omega$ turbulence model, $d=\sqrt{k} / \beta \omega$. This replaces the length scale expression in the dissipative source term of the transport equation of the turbulence kinetic energy $k$.

It has been demonstrated that the DES approach leads to URANS in the near wall region and to a subgrid LES modelling in the farther region, where the vortices detachment occurs. DES is less dissipative than URANS thanks to the choice of the lengthscale that allows increasing the dissipation term in the turbulence kinetic energy equation comparing to URANS. 


\section{3. $\quad$ Delayed detached eddy simulation (DDES)}

To avoid a transition from URANS to LES in the boundary layer that could produce non physical artefacts, Spalart et al. (2006) introduced a modification of the $r_{d}$ parameter of the Spalart-Allmaras model, involved in near-wall damping, as follows :

$$
r_{d}=\frac{\nu+\nu_{t}}{S_{i j} \kappa^{2} d^{2}}
$$

The definition of terms and constants can be found in appendix A. By adding the viscosity $\nu$ on the numerator as a small parameter (in high Reynolds number flows), it is ensured that $r_{d}$ remains away from 0 in the near wall regions. Therefore, a damping function has been suggested : $f_{d}=1-\tanh \left[\left(8 r_{d}^{3}\right)\right]$ which is 1 away from the wall an 0 in the near wall regions where $r d<<1$. Finally, the turbulence length scale becomes :

$$
\tilde{d}=d-f_{d} \max \left(0, d-C_{D E S} \Delta\right) .
$$

If $f_{d}=0, \tilde{d}=d$ which yields to RANS modelling and if $f_{d}=1, \tilde{d}=\min \left(d, C_{D E S} \Delta\right)$ which yields to the classical DES modelling (Spalart et al., 1997). The boundary layer is then shielded from a transition from URANS to LES and this transition is moved farther away from the wall in case of an excessive mesh refinement.

\section{Physical analysis of the dynamic stall around a pitching airfoil at $10^{5}$ and $10^{6}$ Reynolds numbers}

The term dynamic stall usually refers to unsteady separation and stall phenomena on airfoils that are forced to execute time dependant motion. If the angle of attack oscillates around a mean value of the order of the static stall angle, a large hysteresis cycle develop concerning the aerodynamic forces and moment coefficients. Indeed, during the upstroke motion, the effect of adversure pressure gradient is limited, leading to a dynamic stall angle which largely exceeds the static one. The aerodynamics coefficients are higher than their static counterparts. During the downstroke part of the motion, the effect of adverse pressure gradient is reinforced, leading to a reattachment incidence angle which is lower than in the static case.

\subsection{Description of the test cases and numerical methodology}

In both test cases of this study, the airfoils perform a sinusoidal pitching motion which is described in detail in table 1. Turbulence models used for Berton et al test case are Spalart and Allmaras and $k-\varepsilon$ Chien for

Table 1

\begin{tabular}{|c|c|c|}
\hline Experiment & Berton et al. (2002) & McAlister et al. (1978) \\
\hline Reynolds number (based on chord length) & $1.10^{5}$ & $1.10^{6}$ \\
\hline Mean incidence & $12^{\circ}$ \\
\hline Pitch amplitude & $6^{\circ}$ & $10^{\circ}$ \\
\hline Reduced oscillation frequency & 0.188 & 0.1 \\
\hline
\end{tabular}

Physical parameters of Berton et al test case and Mc Alister et al test case.

two-dimensional computations as well as DDES $k-\omega$ SST (Menter, 1993) for three-dimensionnal computations. For the McAlister et al. test case, the turbulence models used are $k-\omega \mathrm{SST}, k-\varepsilon$ Chien and $k-\varepsilon$ OES for twodimensional computations. Three-dimensional computations have not been carried out in this test case because of the high computational time needed in respect of a lower reduced pitching frequency. The mesh used for the Berton et al test case is a $C$ topology mesh with 256 nodes in the $I$ direction, 80 nodes in the $J$ direction and 40 nodes in the $K$ direction for three dimensional computations. The mesh refinement near-wall allows a $y^{+}$of an order of 1 in the attached flow region. For the Mc Alister et al test case, an $O$ topology mesh is used with 285 nodes in the $I$ direction and 185 nodes in the $J$ direction. The mesh refinement allows a $y^{+}$less than one near the wall.

The flow solver is the structured NSMB (Navier Stokes Multi Blocks) code used by the NSMB consortium in which the research group of the authors participates. A detailed description of NSMB, including high-order 
numerical schemes (implicit and explicit) as well as various turbulence closures (standard and advanced URANS), LES and DES can be found in Vos et al. (1998). In the present study, for both test case, the temporal scheme is an implicit backwards second order with dual time stepping. The space discretisation scheme is th upwind-Roe third order with MUSCL fluxes evaluation (van Leer). An adaptive time-step has been used, allowing a CFL number inferior to 1 in the inner loop and inferior to 3 on the outer time-step. The mesh movement in respect of the pitching airfoil is achieved by means of the Arbitrary Lagrangian and Eulerian approach (ALE). The NSMB solver has been used in its full parallelized version for MPI architectures. The super computing centers CINES (Centre Inter-universitaire National d'Enseignement Supérieur), IDRIS (Institut de Recherche en Informatique et Calcul Scientifique), GRID-5000 and CALMIP (Calcul Midi-Pyrennées) have been used for the present computations, providing the CPU allocation.

\subsection{Simulation of the Berton et al test case}

\subsubsection{Two dimensional simulations}

The flow topology is qualitatively in agreement with the experiment. Fig. 1 shows the isovorticity fields for 10 different incidence values. From $7.2^{\circ}$ to $12^{\circ}$ upstroke, the flow remains attached to the profile. From $14.4^{o}$ to $16.8^{\circ}$ the birth of the leading edge vortex can be observed, as well as its progressive growth until it is shed at the maximum incidence. This structure is immediately followed by a trailing edge vortex that also occurs at the maximum incidence. From $18^{\circ}$ to $9.6^{\circ}$ downstroke, the flow is fully stalled and reattachment begins for $7.2^{\circ}$ of incidence downstroke. The above mentioned vortex formation and shedding corresponds to the undulations appearing in the hysteresis loops.

The turbulence models tested display a different behaviour. As seen on Fig. 2, the Spalart and Allmars model over-estimates the maximum lift and drag coefficient, as well as the area under the hysteresis loop. This behaviour is probably due to an excessive dissipative character, which is confirmed by the underestimation of the adverse pressure gradient at high incidence leading to a delay on the prediction of dynamic stall.

The $k-\varepsilon$ Chien model also over-estimates the maximum lift and drag coefficient and the area under the loop but in a less critical way than the Spalart-Allmaras model. This can be explained by a less dissipative character than the previous model. However, the dynamic stall is still enhanced by the beginning of the downstroke phase, showing again an underestimation of the adverse pressure gradient.

The two URANS models tested have shown an over-estimation of the lift coefficient and large lift oscillation during the dowstroke phase which are due to an inhibition of the flow in the third dimension because of the two dimensional computation. Therefore, we can observe that the oscillation amplitude diminishes with the decrease of incidence and the Strouhal number of the vortex shedding is found at 0.48 a value that is close to the experimental one for a static stalled airfoil (Hoarau et al., 2003). This means that the vortices observed are shed on a natural frequency of the flow.

As a conclusion in this part, the $k-\varepsilon$ Chien model provides the best results comparing to Spalart and Allmaras model for the case of a Reynolds $10^{5}$ pitching airfoil. Another source of difference with the experiment is the transitional charter of the boundary layer in the experiment. As the three model examined use the hypothesis of a fully turbulent boundary layer, this partly explain the discrepancy between the experiment and the computations.

\subsubsection{Three dimensional simulations}

The three dimensional computations have shown a practically two-dimensional behaviour during the upstroke part of the motion but a fully three dimensional behaviour during the downstroke phase (Fig. 3). The dynamical increase of incidence is found to enhance two dimensional character of the flow. On the contrary, the decreasing incidence during the downstroke phase of the wing motion reinforces the adverse pressure gradient, producing a fully stalled and highly three-dimensional flow around the airfoil.

Comparing to two-dimensional computations, the hysteresis loops are improved and are able to produce the decrease of the large lift oscillation during the downstroke phase. Therefore, the three dimensional computations show a significant improvement of the results. Considering that for the maximum and minimum incidence of the pitching motion the flow is two dimensional, the extreme lift and drag coefficient are not affected by the threedimensional effects. For this reason, lift and drag coefficient are the same during the upstroke phase in 2-D and 3-D. 


\subsection{Simulation of the McAlister et al test case}

To avoid the transitional boundary layer, computations at a Reynolds number of $10^{6}$ are performed according to the experimennt of McAlister et al. (1978). As seen on Fig. 5 the turbulence models, $k-\omega, k-\epsilon$ Chien and $k-\epsilon$ OES indicate similar behaviour.

The $k-\varepsilon$ Chien provides results close to the experiment with a good prediction of lift, a small overprediction of drag but a large delay on the predicted stall angle. This means that the model has an excessive dissipative character that prevents from a correct prediction of the adverse pressure gradient leading to stall. As a result, its behaviour is forced by the movement of the structure.

The $k-\omega$ SST gives the most accurate results with a good estimation of the stall angle and good prediction of the lift and drag coefficient during the upstroke motion. However, this model gives the less smooth character which could be due to the addition of the SST limiter that creates abrupt reduction of the eddy viscosity.

The $k-\epsilon$ OES modelling predicts the best angle of dynamic stall but overestimate the lift and drag coefficients.

The three models are over-estimates the lift coefficient during the downstroke motion. The undulations appearing in the lift and drag hysteresis are due to the coherent vortex shedding. This vortex shedding is more pronounced in the 2-D simulations, because of flow confinement induced by the two-dimensional approximation.

\section{Conclusion and prospects}

In this study, two- and three-dimensional simulations have been performed comparing the behaviour of various URANS and hybrid turbulence modelling approaches on the flow around a pitching airfoil.

Advanced turbulence models like OES and SST modelling have shown better results than classical URANS models for the occurence of dynamic stall prediction.

The $10^{5}$ Reynolds number computations have shown the need of a transition model.

Finally, three dimensional computations have shown an improvement on the downstroke phase allowing the decrease of the coherent structure amplitude comparing to 2-D computations.

However, the two-dimensional study is useful for fast pre-design use, because they are able to capture a significant part of the coherent structures dynamics in a good approximation comparing to the three-dimensional simulations. 


\section{A Description of the Spalart and Allmaras one equation turbulence model}

The Spalart and Allmaras model (Spalart and Allmaras, 1992) is a one equation turbulence model. The transport equation for eddy viscosity is made under dimensional analysis assumptions.

The eddy viscositytransport equation is given by

$$
\frac{\partial \tilde{\nu}}{\partial t}+\tilde{u_{j}} \frac{\tilde{\nu})}{\partial x_{j}}=P+D_{I F F}+D_{E S}
$$

where $P, D_{I F F}$ and $D_{E S}$ are respectively production, dissipation and destruction terms of $\tilde{\nu}$ expressed as

$$
\begin{gathered}
P=c_{b 1} \tilde{S} \tilde{\nu} \\
D_{I F F}=\frac{1}{\rho}\left[\frac{\partial}{\partial x_{j}}\left((\nu+\tilde{\nu}) \frac{\partial \tilde{\nu}}{\partial x_{j}}\right)+c_{b 2} \frac{\partial \tilde{\nu}}{\partial x_{j}} \frac{\partial \tilde{\nu}}{\partial x_{j}}\right], \\
D_{E S}=c_{\omega 1} f_{\omega}\left(\frac{\tilde{\nu}}{d}\right)^{2} .
\end{gathered}
$$

The eddy viscosity is then defined as follows :

$$
\nu_{t}=\tilde{\nu} f_{v 1}
$$

To ensure that $\tilde{\nu}$ is equal to $\kappa y u_{\tau}$ in the logarithmic part of the boundary layer, the damping function $f_{v 1}$ is defined as

$$
f_{v 1}=\frac{\chi^{3}}{\chi^{3}+c_{v 1}^{3}}
$$

where $\chi=\tilde{\nu} / \nu$.

However, $S$ is modified in $\tilde{S}$ in order to maintain a correct behaviour in the logarithmic part of the boundary layer $\left(\tilde{S}=u_{\tau} / \kappa y\right)$ :

$$
\tilde{S}=\sqrt{2 \Omega_{i j} \Omega_{i j}} f_{v 3}+\frac{\tilde{\nu}}{\kappa^{2} d^{2}} f_{v 2}
$$

with $\Omega=\frac{1}{2}\left(\frac{\partial \tilde{u_{i}}}{\partial x_{j}}-\frac{\partial \tilde{u_{j}}}{\partial x_{i}}\right), f_{v 2}=1-\frac{\chi}{\chi+f_{v 1}}$ and $f_{v 3}=1$. Finally, in order to obtain a decrease of the destruction part outside the boundary layer, the function $f_{\omega}$ i introduced such that :

$$
f_{\omega}(g)=g\left(\frac{1+c_{\omega 3}^{6}}{g^{6}+c_{\omega 3}^{6}}\right)^{\frac{1}{6}},
$$

where $g$ acts as a limiter, avoiding $f_{\omega}$ to become to high and is expressed as : $g=r+c_{\omega 2}\left(r^{6}-r\right)$ with $r=\frac{\tilde{\nu}}{\tilde{S} \kappa^{2} d^{2}}$. $r$ and $f_{\omega}$ are both equal to 1 in the logarithmic part of the boundary layer and decrease outside.

Constants of the model are $: c_{b 1}=0.1355, c_{b 2}=0.622, \sigma=\frac{2}{3}, \kappa=0.41, c_{\omega 1}=\frac{c_{b 1}}{\kappa^{2}}+\frac{1+c_{b 2}}{\sigma}, c_{\omega 2}=0.3, c_{\omega 3}=2$ et $c_{v 1}=7.1$. The Spalart and Allmaras model is considered as a good compromise between algebraic models and two equation models. This model has become very popular due to its robustness and reasonable predictive efficiency in a wide number of aerodynamic flows. 


\section{References}

Akbari, M. H., Price, S. J., May 2003. Simulation of dynamic stall for a NACA 0012 airfoil using a vortex method. Journal of Fluids and Structures 17, 855-874.

Benyahia, A., Berton, E., Favier, D., Maresca, C., Badcock, K., Barakos, G., 2003. Detailed evaluation of cfd predictions against lda measurement for flow $\mathrm{n}$ an aerofoil. In: Proceedings of the Integrating CFD and Experiment symposium, September 8-9, 2003, Glasgow, United Kingdom.

Berton, E., Allain, C., Favier, D., Maresca, C., 2002. Experimental methods for subsonic flow measurements. Notes on Numerical Fluid Mechanics and Multidisciplinary Design 81, 251-260, dedicated volume in "Progress in Computational Flow-Structure Interaction".

Berton, E., Favier, D., Maresca, M., 1997. Embedded l.v. methodology for boundary-layer measurements on oscillating models. AIAA Paper 97-1832.

Bouhadji, A., Bourdet, S., Braza, M., Hoarau, Y., Rodes, P., G.Tzabiras, 2002. Turbulence modelling of unsteady flows with a pronounced periodic character. Notes on Numerical Fluid Mechanics and Multidisciplinary Design 81, 87-96, dedicated volume in "Progress in Computational Flow-Structure Interaction".

Bourdet, S., Braza, M., Hoarau, Y., Akoury, R. E., Ashraf, A., Harran, G., Chassaing, P., Djeridi, H., 2007. Prediction and physical analysis of unsteady flows around a pitching airfoil with the dynamic mesh approach. European Journal of Computational Mechanics 16.

Bourguet, R., Braza, M., Perrin, R., Harran, G., mai 2007. Anisotropic eddy-viscosity concept for strongly detached unsteady flows. AIAA Journal 45, 1145-1149.

Braza, M., Perrin, R., Hoarau, Y., 2006. Turbulence properties in the cylinder wake at high Reynolds number. Journal of Fluids and Structure 22, 757-771.

Cantwell, B., Coles, D., 1983. An experimental study of entrainment and transport in the turbulent near wake of a circular cylinder. Journal of Fluid Mechanics 136, 321-374.

Chandrasekhara, M., Carr, L., 1990. Flow visualisations studies of the Mach number effets on dynamic stall. Journal of Aircraft 27, 516-522.

Chien, K., 1982. Predictions of channel and boundary-layer flows with a low-Reynolds number turbulence model. AIAA Journal 20, 33-38.

Dervieux, A., Braza, M., Dussauge, J., 1998. Computation and comparison of efficient turbulence models for aeronautics. In: Notes on Numerical Fluid Mechanics. Vol. 65.

Haase, W., Selmin, V., Winzell, B., 2002. Progress in computational flow-structure interaction, results of the project UNSI, supported by the european union 1998 - 2000. Notes on Numerical Fluid Mechanics and Multidisciplinary Design 81.

Hoarau, Y., Braza, M., Tzabiras, G., Allain, C., Berton, E., Favier, D., Maresca, C., 2002. Prediction of turbulent unsteady aerodynamic flows with a pronounced periodic character. In: IUTAM Symposium on Unsteady Separated Flows. Toulouse, France.

Hoarau, Y., Braza, M., Ventikos, Y., Faghani, D., Tzabiras, G., 2003. Organized modes and the three-dimensional transition to turbulence in the incompressible flow around a naca0012 wing. Journal of Fluid Mechanics 496, 63-72.

Jin, G., Braza, M., 1994. A two-equation turbulence model for unsteady separated flows around airfoils. AIAA Journal 32, $2316-2320$.

Launder, B., Reece, G., Rodi, W., 1975. Progress in the development of a Reynolds stress turbulence closure. Journal of Fluid Mechanics 68, 537-566.

McAlister, K., Carr, L., McCroskey, W., 1978. Dynamic stall experiments on the NACA0012 airfoil. Technical Paper TP 1100, NASA.

McCroskey, W. J., 1981. The phenomenon of dynamic stall. NASA report : NASA/TM-81264.

McCroskey, W. J., 1982. Unsteady airfoils. Annual Revue of Fluid Mechanics 14, 285-311.

Menter, F. R., 1993. Zonal two equation $k-\omega$ turbulence models for aerodynamics flows. AIAA Paper 93-2906.

Piziali, R. A., 1993. An experimental investigation of 2D end 3D oscillating wing aerodynamics for a range of angle of attack including stall. NASA technical memorandum, no 4632. Ames, CA:NASA 4632, Chalmers University of Technology.

Rung, T., Bunge, U., Schatz, M., Thiele, F., 2002. Restatement of the spalart-allmaras eddy-vicosity model in a strain adaptative formulation. AIAA Journal 41(7), 1396-1399.

Spalart, P., Deck, S., Shur, M., Squires, K., Strelets, M., Travin, A., 2006. A new version of detached eddy simulation, resistant to ambiguous grid densities. Journal of Theoretical and Computational Fluid Dynamics 20, $181-195$.

Spalart, P., Jou, W.-H., Strelets, M., Allmaras, S. R., 1997. Comments on the feasibility of LES for wings, and on a hybrid RANS/LES approach. In: First AFOSR International Conference on DNS/LES, Aug. 4-8. Ruston, Louisiana.

Spalart, P. R., Allmaras, S. R., 1992. A one equation turbulence model for aerodynamics flows. AIAA paper 92-0439.

Travin, A., Shur, M., Strelets, M., Spalart, P., 2000. Detached-Eddy Simulations past a Circular Cylinder. Flow, Turbulence and Combustion 63, 293-313.

Tsang, K. K. Y., So, R. M. C., Leung, R. C. K., Wang, X. Q., Jan. 2008. Dynamic stall behavior from unsteady force measurements. Journal of Fluids and Structures 24, 129-150.

Vos, J., Chaput, E., Arlinger, B., Rizzi, A., Corion, A., 1998. Recent advances in aerodynamics inside the nsmb (navier stokes multi blocks) consortium. AIAA Paper 1998-0802.

Wallin, S., Johansson, A. V., 2000. An explicit algebraic Reynolds model for incompressible and compressible turbulent flows. Journal of Fluid Mechanics 403, 89-132. 


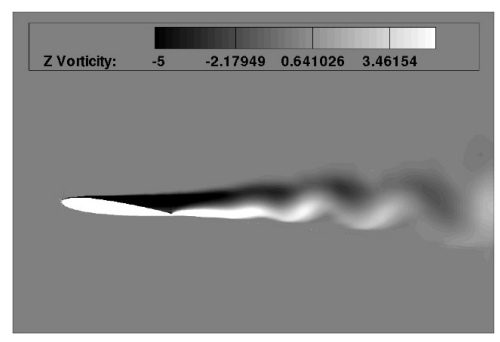

$\alpha=7.2^{\circ}$ upstroke

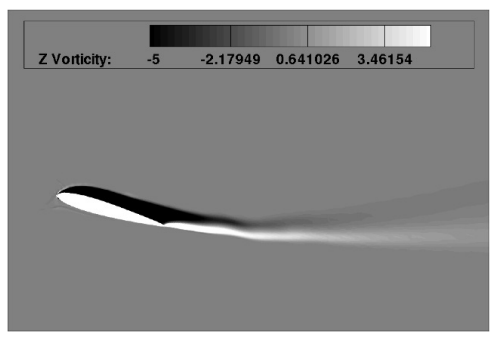

$\alpha=14.4^{\circ}$ upstroke

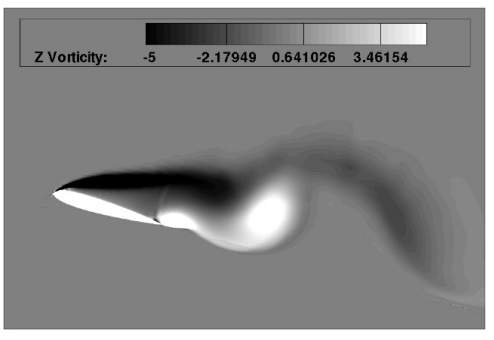

$\alpha=14.4^{\circ}$ downstroke

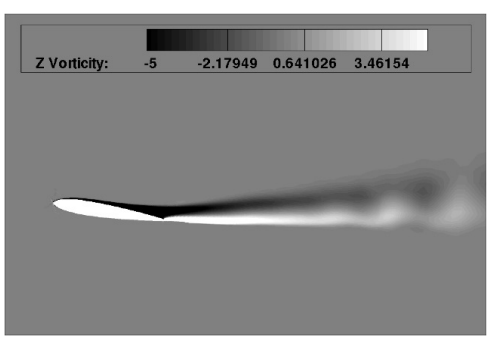

$\alpha=9.6^{\circ}$ upstroke

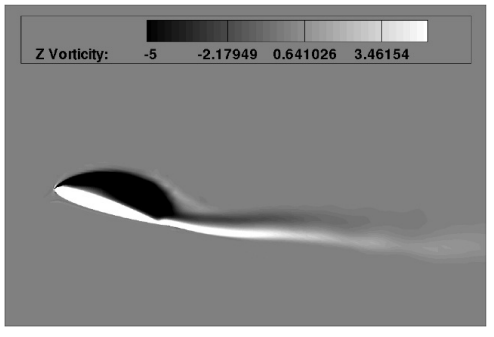

$\alpha=16.8^{\circ}$ upstroke

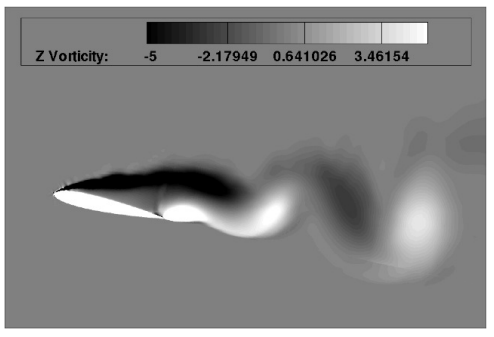

$\alpha=12^{\circ}$ downstroke

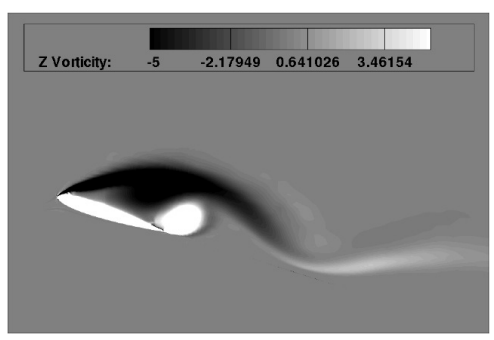

$\alpha=16.8^{\circ}$ downstroke

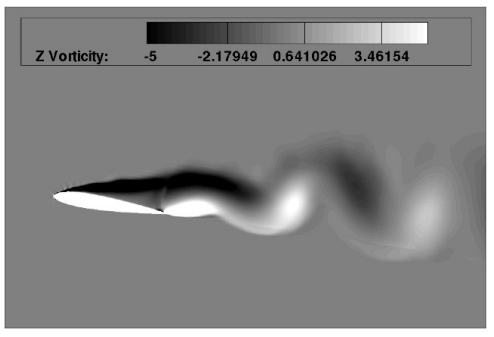

$\alpha=9.6^{\circ}$ downstroke

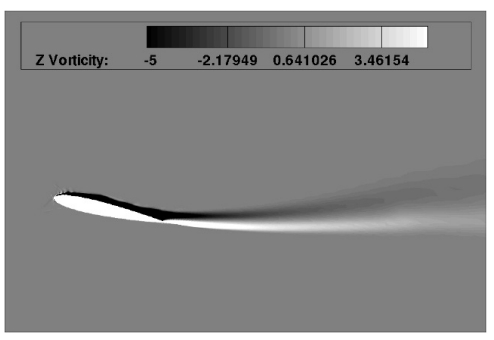

$\alpha=12^{\circ}$ upstroke

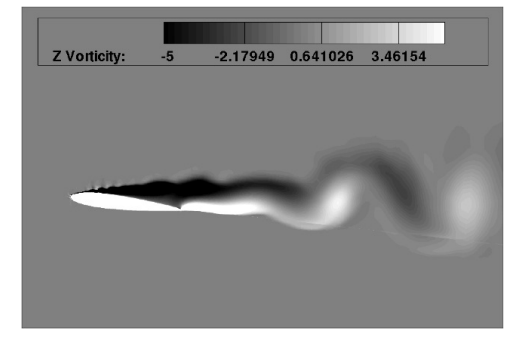

$\alpha=7.2^{\circ}$ downstroke

Fig. 1. Isovorticity field for a pitching cycle using $k-\varepsilon$ Chien modelling 

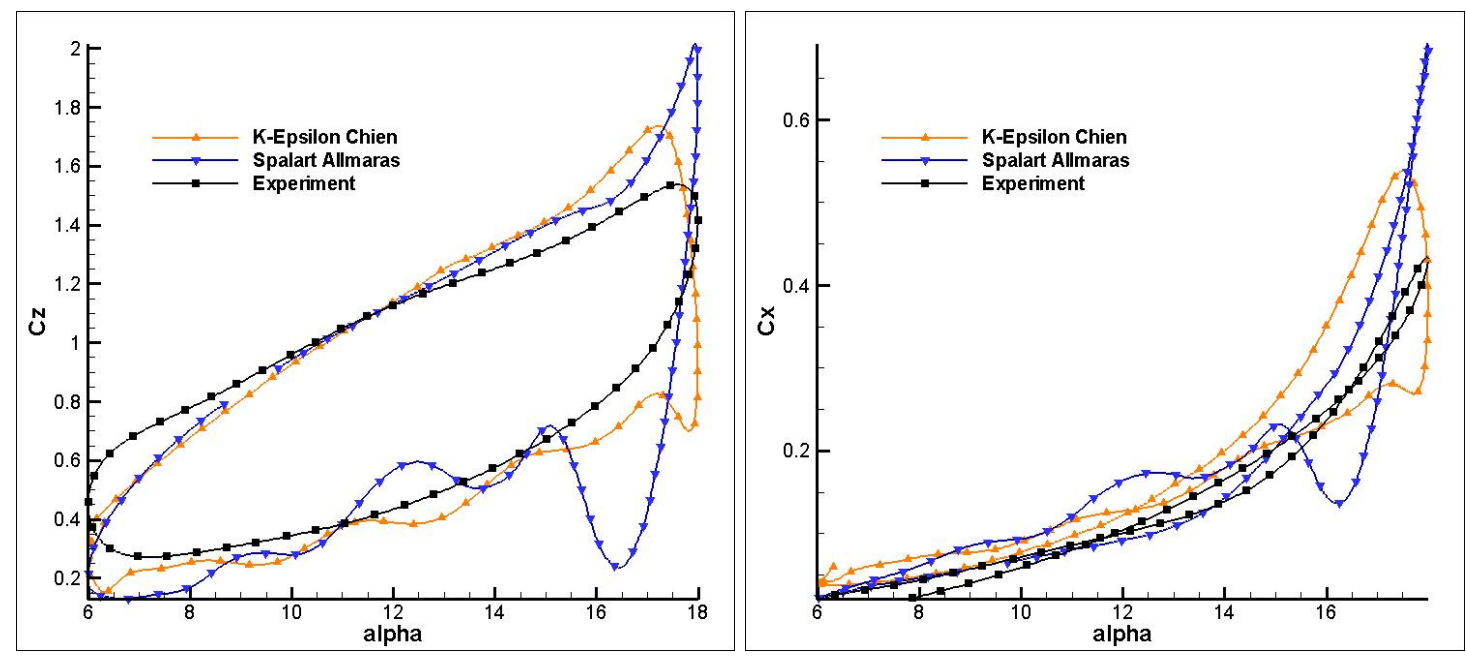

Fig. 2. Hysteresis loops obtained with 2-D computations on lift and drag coefficient average on five pitching cycle and compared to experimental results 


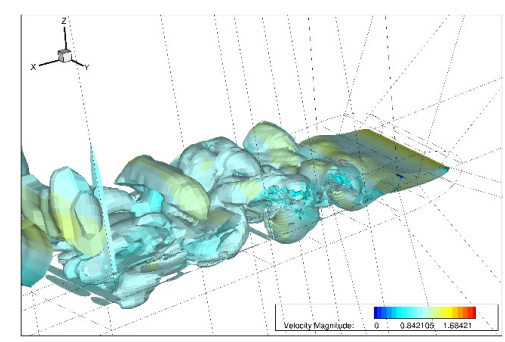

$\alpha=6^{\circ}$

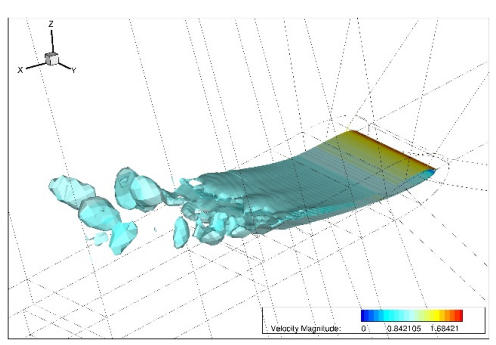

$\alpha=12^{\circ}$ upstroke

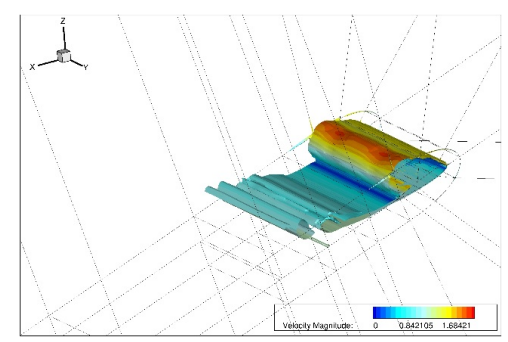

$\alpha=18^{\circ}$

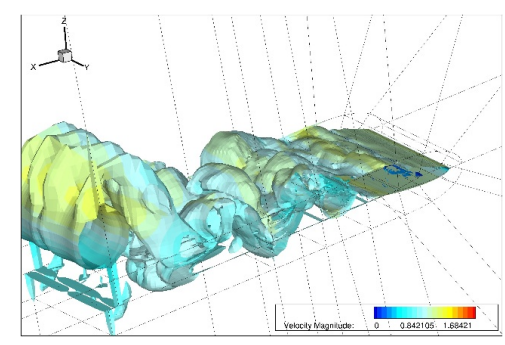

$\alpha=12^{\circ}$ downstroke

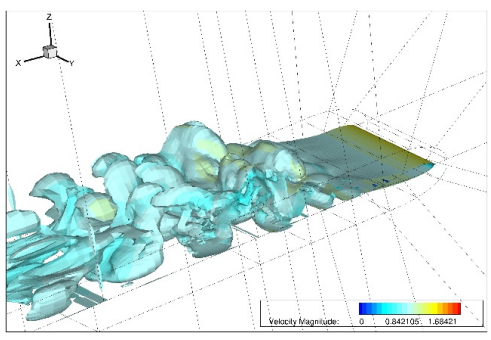

$\alpha=8^{\circ}$ upstroke

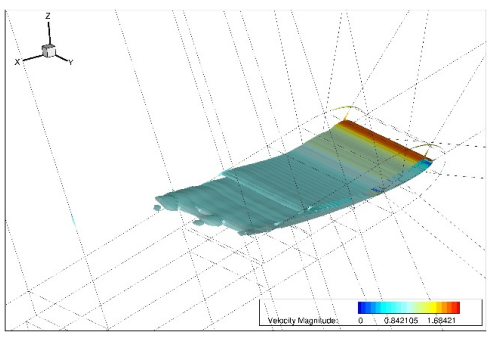

$\alpha=14^{\circ}$ upstroke

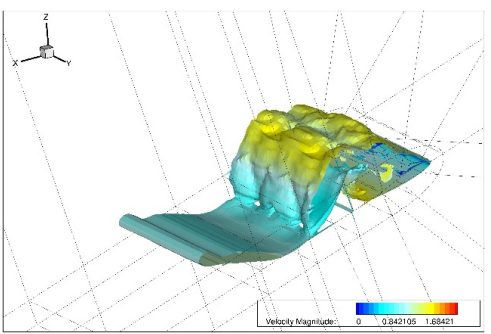

$\alpha=16^{\circ}$ downstroke

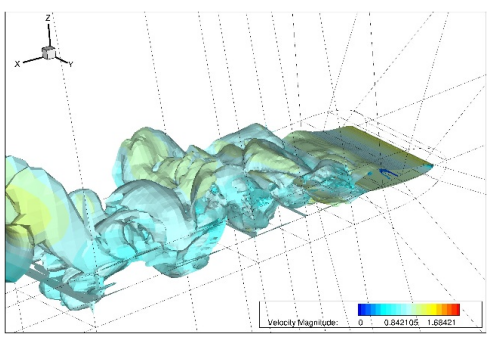

$\alpha=10^{\circ}$ downstroke

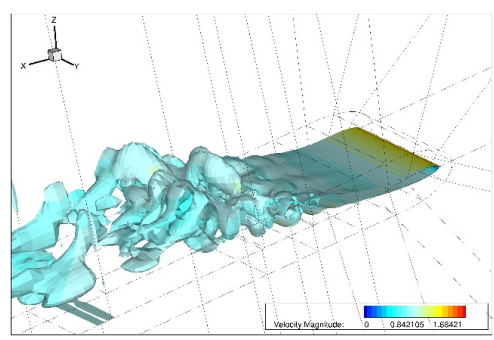

$\alpha=10^{\circ}$ upstroke

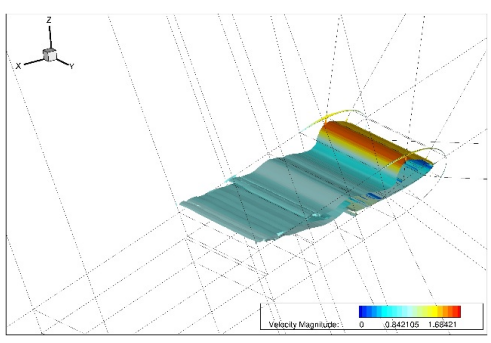

$\alpha=16^{\circ}$ upstroke

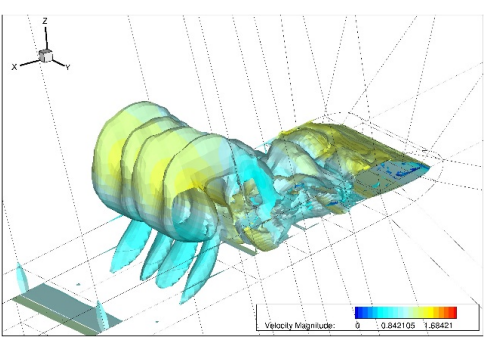

$\alpha=14^{\circ}$ downstroke

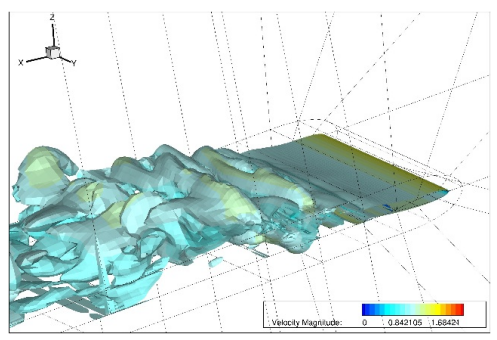

$\alpha=8^{\circ}$ downstroke

Fig. 3. Vorticity isosurfaces at 1, colormap as a function of velocity magnitude between 0 and 2 on a pitch cycle. 

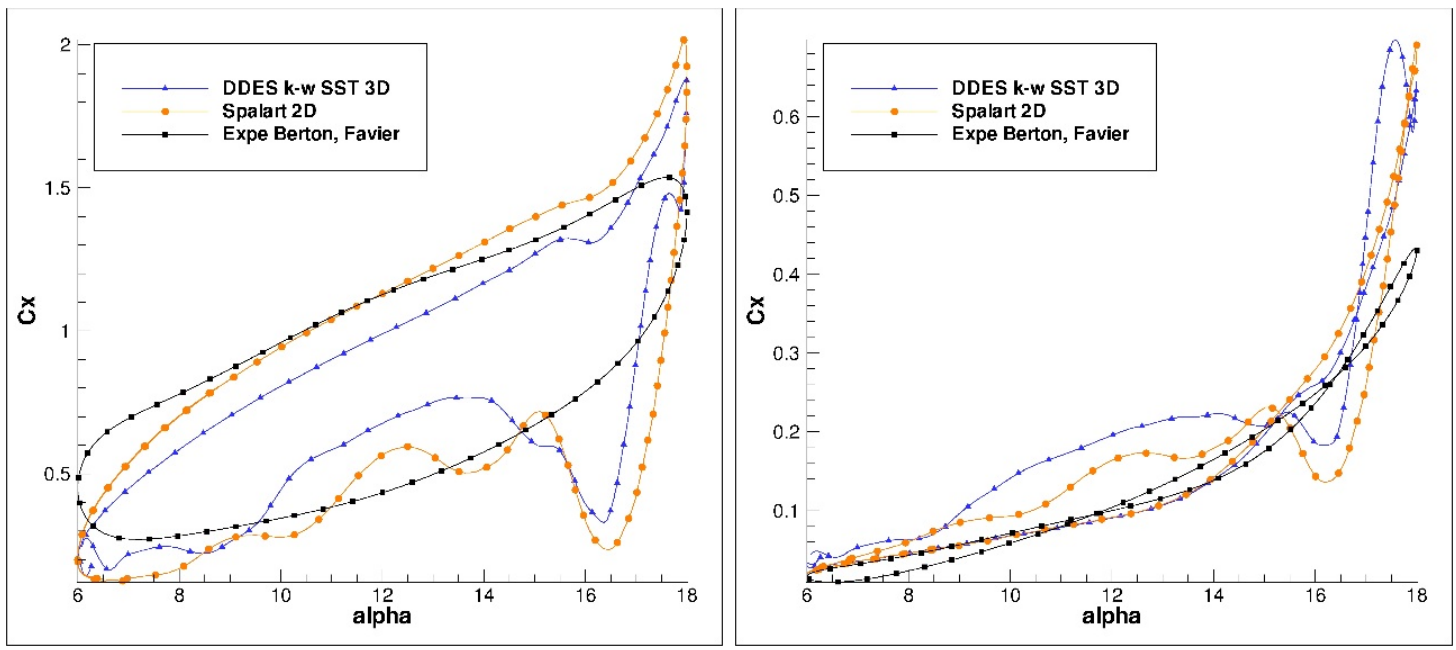

Fig. 4. Hysteresis loops obtained with 3-D computations on lift and drag coefficient and compared to experimental results and 2-D computations with Spalart and Allmaras model

Fig. 5. Hysteresis loops obtained with 2-D computations on lift and drag coefficient and compared to experimental results 